\title{
Kappa Opioid Receptors on Dopaminergic Neurons Are Necessary for Kappa-Mediated Place Aversion
}

\author{
Vladimir I Chefer*,', Cristina M Bäckman', Eduardo D Gigante' and Toni S Shippenberg' \\ IIntegrative Neuroscience Section, Integrative Neuroscience Branch, National Institute on Drug Abuse, Baltimore, MD, USA
}

\begin{abstract}
Kappa-opioid receptor (KOR) agonists have dysphoric properties in humans and are aversive in rodents. This has been attributed to the activation of KORs within the mesolimbic dopamine (DA) system. However, the role of DA in KOR-mediated aversion and stress remains divisive as recent studies have suggested that activation of KORs on serotonergic neurons may be sufficient to mediate aversive behaviors.

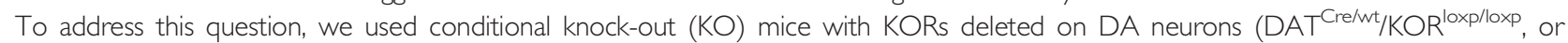
DATCre-KOR KO). In agreement with previous findings, control mice (DAT ${ }^{\mathrm{Cre} / w t} / K O \mathrm{R}^{\mathrm{wt} / \mathrm{wt}}$ or WT) showed conditioned place aversion (CPA) to the systemically administered KOR agonist U69,593. In contrast, DATCre-KOR KO mice did not exhibit CPA with this same agonist. In addition, in vivo microdialysis showed that systemic U69,593 decreased overflow of DA in the nucleus accumbens (NAc) in WT mice, but had no effect in DATCre-KOR KO mice. Intra- ventral tegmental area (VTA) delivery of KORs using an adeno-associated viral gene construct, resulted in phenotypic rescue of the KOR-mediated NAc DA response and aversive behavior in DATCre-KOR KO animals. These results provide evidence that KORs on VTA DA neurons are necessary to mediate KOR-mediated aversive behavior. Therefore, our data, along with recent findings, suggest that the neuronal mechanisms of KOR-mediated aversive behavior may include both dopaminergic and serotonergic components.

Neuropsychopharmacology (2013) 38, 2623-263I; doi:I0.1038/npp.2013.171; published online 7 August 2013
\end{abstract}

Keywords: Kappa-opioid receptor; conditioned place aversion; dopamine.

\section{INTRODUCTION}

Opioid systems are implicated in numerous neurobiological functions, including nociception, reward, and stress. However, the physiological roles and mechanisms of action for opioid receptor subtypes have not yet been clearly defined, particularly with regard to kappa-opioid receptors (KORs). In contrast to mu- or delta-opioid agonists, KOR agonists have dysphoric and psychotomimetic properties in humans (Pfeiffer et al, 1986), and are aversive in rodents (Shippenberg et al, 1993; Knoll et al, 2011; Zhang et al, 2005; Mucha and Herz, 1985). Thus, microinjections of KOR agonists into the ventral tegmental area (VTA) or nucleus accumbens (NAc) produce conditioned place aversion (CPA; Bals-Kubik et al, 1993a) and decrease dopamine (DA) overflow, whereas 6-OHDA lesions or NAc D1 receptor blockade abolish CPA produced by systemically administered KOR agonists (Shippenberg et al, 1993). Therefore, these data support the hypothesis that the aversive properties of KOR agonists are mediated by a negative modulation of the mesolimbic DA system (Shippenberg et al, 1993).

*Correspondence: Dr VI Chefer, Behavioral Neuroscience Section, NIDA/IRP, 25I Bayview Boulevard, RM 8A721, Baltimore, MD 21224 , USA, Tel: 443740 2869, Fax: 4437402827 ,

E-mail: vchefer@intra.nida.nih.gov

Received 25 April 20 13; revised 21 June 2013; accepted 21 June 20 I3; accepted article preview online 18 July 2013
In contrast to these prior data, recent results from DA-deficient mice have shown that CPA to the KOR agonist U50,488 is unaffected (Land et al, 2009), implying that the previously demonstrated reduction in DA release by KOR agonists may not be necessary for KOR-induced aversion. This recent study (Land et al, 2009) also demonstrated that activation of KORs either pharmacologically or by endogenous opioids released by stressful stimuli may regulate a serotonergic projection from the dorsal raphe nucleus (DRN) to the NAc and that these projections are both necessary and sufficient for KOR CPA. However, the role of DA was not specifically examined in this study, and the interpretation of data obtained from constitutive knock-out (KO) mice may have been complicated by compensatory adaptations in neuronal circuitry during development. In addition, the DA-deficient mice used by Land et al (2009) were hypoactive and received caffeine before testing, which may also lead to unintended interactions.

The aim of the present study was to address this existing contradiction. We sought to determine if KORs located on DA neurons and modulation of dopaminergic neurotransmission are necessary for the development of a CPA to a systemically administered KOR agonist using conditional KO mice with KORs deleted on DA neurons. Here we report that KORs on DA cells are indeed necessary for kappamediated aversive behavior. 


\section{MATERIALS AND METHODS}

\section{Animals}

Male C57BL/6 (Charles River Laboratories) or transgenic mice on a C57BL/6 background were used in these experiments. For conditional KO of KORs in DA neurons, we used a dopamine transporter (DAT) promoter-driven Cre transgenic mouse line, Slc6a3 ${ }^{\mathrm{Cre}}$ (DAT $\left.{ }^{\mathrm{Cre}}\right)$. DAT ${ }^{\mathrm{Cre}}$ (Backman et al, 2006) that were crossed with $\mathrm{KOR}^{\operatorname{loxp}}$ (Dr Jennifer Whistler) mice to obtain regional $\mathrm{KO}$ (DAT ${ }^{\mathrm{Cre} / \mathrm{wt}}$ $\mathrm{KOR}^{\text {loxp/loxp }}$ or DATCre-KOR KO) and control mice $\left(\mathrm{DAT}^{\mathrm{Cr} / \mathrm{wt}} \mathrm{KOR}^{\mathrm{wt} / \mathrm{wt}}\right.$ or WT). Briefly, KORloxp mice were generated by flanking Exon 2 (containing the start codon) of the Oprk1 gene with loxp sequences. DATCre mice were generated by inserting an internal ribosome entry-linked Cre recombinase gene downstream from the stop codon of the endogenous Slc6a3 gene. Both mouse strains were crossed for at least 10 generations with C57BL/6J background mice at the time of crossbreeding, and therefore were considered congenic. Mice $(20-30 \mathrm{~g})$ were housed 2-4 per cage before surgery, and singly housed after surgery in facilities accredited by the American Association for the Accreditation of Laboratory Animal Care. All experimental protocols were approved by the NIH/NIDA Intramural Research Program Institutional Care and Use Committee. Mice were maintained at a constant temperature $\left(22 \pm 2{ }^{\circ} \mathrm{C}\right)$ on a 12 -h light/dark cycle (light from 0700 to 1900 hours). All animal procedures were performed during the light cycle.

\section{Genomic DNA Analysis of DATCre-KOR KO Mice}

To confirm if Oprk1 exon 2 deletion was specific to DATexpressing regions, primers specific to the recombination event were developed (F: 5'-GCTAGTGCTTCTTGGGGTTG-3' and B: $5^{\prime}$-CCTGCAGGAAGTACCAGAGC-3'). Genomic DNAs from three KO animals were prepared from the following brain regions: olfactory bulb, striatum, ventral midbrain, cortex, and cerebellum. For ventral midbrain dissections, coronal cuts were made at the anterior and posterior boundaries of the mammillary nucleus. Dissection of the ventral midbrain region containing the substantia nigra (SN) and VTA was facilitated by removing the mammillary nucleus on the ventral surface and overlying cortex on the dorsal surface. A block containing the SN/VTA was then dissected. To further ensure specificity of Cre-mediated recombination, lung, heart, liver, gut, kidney, and muscle tissues were dissected and DNA extracted for PCR analysis. In addition, the recombination band was isolated from the agarose gel and sequence verified.

\section{Double In-Situ Hybridization (ISH)}

To further verify Cre-mediated recombination in DATCreKOR mice, and to verify AAV2-CAG-KOR injections, we used dual fluorescent ISH. WT $(n=3)$, DATCre-KOR KO $(n=3)$, and DATCre-KOR KO mice injected with AAV2GAG-KOR $(n=3)$ were euthanized via $\mathrm{CO}_{2}$ exposure. Brains were extracted and fresh frozen in dry ice. Brain sections ( $10 \mu \mathrm{m}$ in thickness) containing the ventral midbrain were subjected to dual fluorescent ISH using RNAscope (Advanced Cell Diagnostics) following the manufacturer's protocol. Sequences for probe generation were as follows: KOR probe nt 176-481 of NM_001204371 (correspond to loxp flanked region in $\mathrm{KOR}^{\mathrm{loxP}}$ mice) and DAT probe nt 1486-2525 of NM_010020.3. To verify AAV2-CAGGFP injections, sections from DATCre-KOR animals $(n=3)$ were processed for DAT immunocytochemistry as previously described (Backman et al, 2006). Qualitative analyses were performed by evaluating co-localization of DAT and KOR mRNA in 20 randomly selected fields of view $(\times 40)$ per animal corresponding to the $\mathrm{SN}$ and VTA regions from three different WT and DATCre-KOR KO mice.

\section{Surgical Procedures and Drugs}

Mice were anesthetized with ketamine-xylazine $(80-18 \mathrm{mg} / \mathrm{kg}$ i.p.) and implanted with a unilateral microdialysis guide cannula (CMA7; CMA Microdialysis) aimed at the NAc (AP + $1.7 \mathrm{~mm}$; ML: $-0.5 \mathrm{~mm}$; DV: $-4.0 \mathrm{~mm}$; Paxinos and Franklin, 2001). U69,593 was supplied by the Research Technology Branch of NIDA (Rockville, MD). Stock solutions of U69,593 $(10 \mathrm{mM})$ was dissolved in $0.1 \mathrm{~N} \mathrm{HCl}$ and diluted in sterile saline. The $\mathrm{pH}$ of saline-containing drugs was adjusted to 7.2-7.4 using $\mathrm{NaOH}$.

\section{CPA}

Conditioning procedures were carried out using a conditioning apparatus consisting of two discrete compartments $\left(23.2 \times 12.7 \times 12.7 \mathrm{~cm}^{3}\right)$ with black walls and removable floors (Med Associates). The CPA procedure consisted of four phases: habituation, pre-conditioning test (pre-test), conditioning and post-conditioning test (post-test). Locomotor activity was measured during each phase of CPA procedure.

Animals were first habituated to the apparatus by allowing free access to both compartments for $15 \mathrm{~min}$. During habituation, smooth plastic floors (different from the floors used during conditioning) were present in both compartments to prevent latent inhibition (when nonreinforced pre-exposure to a stimulus retards subsequent conditioning (Lubow, 1973)). The following day, for pretest, one compartment was equipped with a mesh floor and the other compartment with grid floor. Mice were allowed to explore both compartments for $15 \mathrm{~min}$ and the time spent in each compartment was measured. Overall initial conditions were unbiased for all groups (Cunningham et al, 2006). During the 6 days of conditioning, animals were injected once a day with either vehicle (saline, $0.1 \mathrm{ml} / 10 \mathrm{~g}$, s.c.) or $\mathrm{U} 69,593(0.32 \mathrm{mg} / \mathrm{kg} ; 0.1 \mathrm{ml} / 10 \mathrm{~g}$, s.c.) and immediately confined to one randomly assigned compartment with either mesh or grid floor for $45 \mathrm{~min}$. The order of drug administration was counterbalanced such that half the animals received U69,593 on the first day of conditioning and the other half on the second day of conditioning. The day after the last conditioning session, mice were allowed access to all compartments and tested once again for preference for $15 \mathrm{~min}$ (post-test). The primary index for determining place conditioning was a significant difference between time spent on the drug-paired floor during the preand the post-test. Conditioning scores represented time spent in vehicle- or drug-paired place during post-test minus that spent in the same place during pre-test. 


\section{Microdialysis Procedures}

Microdialysis experiments were conducted as previously described (Chefer et al, 2004; Chefer et al, 2006; Chefer et al, 2009). Mice were allowed to recover from the surgery for 5-7 days before dialysis. The evening before testing, animals were lightly restrained and a microdialysis probe (CMA/7; $0.24 \times 2 \mathrm{~mm}$; CMA Microdialysis) was manually inserted through the guide cannula. The inlet tubing of the probe was connected to a microinfusion pump (CMA 102) via a dual quartz-lined swivel (Instech) and animals were placed into Plexiglas chambers $\left(40 \times 40 \times 35 \mathrm{~cm}^{3}\right)$. Probes were perfused with aCSF $(145 \mathrm{mM} \mathrm{NaCl}, 2.8 \mathrm{mM} \mathrm{KCl}, 1.2 \mathrm{mM}$ $\mathrm{MgCl}_{2}, 1.2 \mathrm{mM} \mathrm{CaCl}, 5.4 \mathrm{mM}$ glucose, adjusted to $\mathrm{pH}$ 7.2-7.4 using $\mathrm{NaOH}$ ) overnight at a flow rate of $0.3 \mu \mathrm{l} / \mathrm{min}$. The following day, perfusate was replaced with fresh aCSF and the flow rate was changed to $1 \mu \mathrm{l} / \mathrm{min}$. Following a 2 -h equilibration period, six $10 \mathrm{~min}$ baseline samples were collected before perfusion of U69,593 $(1.0 \mu \mathrm{M})$. After a 30 min equilibration period, six consecutive samples were collected during drug perfusion and perfusate was replaced with normal aCSF. Six more samples were collected following a 30 -min equilibration period.

\section{Microinjection Procedures}

Mice were anesthetized with ketamine-xylazine (80-18 mg/kg i.p.) and placed in a stereotactic frame (David Kopf). Small holes were drilled into the skull over the designated areas. Injector cannula (C313, Plastics One) connected to a $50-\mu 1$ Hamilton syringe was lowered bilaterally into the VTA (AP $-3.1 \mathrm{~mm}$; ML: $\pm 0.5 \mathrm{~mm}$; DV: $-4.7 \mathrm{~mm}$ ) for AAV2CAG-KOR $(1 \mu \mathrm{l}$ per site)/AAV2-CAG-GFP ( $1 \mu \mathrm{l}$ per site) microinjections. Compounds were infused at a rate of $0.5 \mu \mathrm{l} / \mathrm{min}$ for $2 \mathrm{~min}$ with the stereotaxic injector (QSI, Stoelting). The injector was left in place for at least $5 \mathrm{~min}$ to allow for diffusion. Following microinjections, incisions were sutured and animals recovered until the experiments started, 20-21 days after intra-VTA infusions of AAV2CAG-KOR/AAV2-CAG-GFP. The AAV2 vectors $(1.2 \times$ $10^{13} \mathrm{GC} / \mathrm{ml}$ ) were obtained from Vector Biolabs and stored at $-80{ }^{\circ} \mathrm{C}$ before use.

\section{Histological Analyses}

After each experiment, animals were deeply anesthetized with pentobarbital and killed by decapitation. The brains were removed and frozen, and the site of probe/injector placement was verified histologically via coronal sections (20 $\mu \mathrm{m}$ thick) using a microtome (model 5030; Hacker Instrument).

\section{Chromatographic Analysis of Brain Microdialysates}

Dialysate DA concentrations were analyzed using highperformance liquid chromatography (HPLC) with electrochemical detection. Aliquots from dialysate samples $(10 \mu \mathrm{l})$ were injected into an Eicom HTEC-500 HPLC system with an integrated amperometric detector, consisting a PP-ODS HPLC column $\left(30 \times 4.6 \mathrm{~mm}^{2}\right.$, inner diameter, C-18, $2 \mu \mathrm{m}$ particulate silica gel) and a HTEC-500 pump (Eicom). The mobile phase consisted of $100 \mathrm{mM} \mathrm{NaH}_{2} \mathrm{PO}_{4}, 1.3 \mathrm{mM}$ EDTA,
$2.0 \mathrm{mM}$ decane-1-sulfonate, $1 \% \mathrm{MeOH}, \mathrm{pH} 6.0$, resulting in a retention time of $2.2-2.5 \mathrm{~min}$ at a pump rate of $0.5 \mathrm{ml} / \mathrm{min}$. Applied potential was set at $+400 \mathrm{mV} v s \mathrm{Ag} / \mathrm{AgCl}$. Concentration of dialysate DA levels were estimated using calibration curves obtained from external standards. Detection limit of DA using these conditions was approximately $50 \mathrm{pM}$. Dialysate samples were analyzed within $24 \mathrm{~h}$ of collection to prevent degradation.

\section{Data Analysis}

Dialysate concentrations were expressed as absolute values $(\mathrm{nM})$ and percentage of the baseline. Repeated-measures ANOVA with one between factor (genotype: WT $v s$ DATCre-KOR KO) and two within factors (drug challenge: baseline $v s \mathrm{U} 69,593$, and time) was used to analyze time course data in microdialysis experiments. Two-way ANOVA or repeated-measures ANOVA with two between factors (genotype and microinjections: vehicle $v s$ nor-BNI or AAV2-CAG-KOR $v s$ AAV2-CAG-GFP) and one within factor (pre-test $v s$ post-test) was used to analyze CPA data. Data are presented as mean \pm standard error of the mean (SEM). The accepted value of significance was $P \leqslant 0.05$.

\section{RESULTS}

Cre-Mediated Recombination is Restricted to the Ventral Mesencephalon and Olfactory Bulb in DATCre-KOR KO Mice and it is Specific to DA Neurons within the VTA and $\mathrm{SN}$

To assess the spatial specificity of Cre-mediated recombination in DATCre-KOR KO animals, we first examined recombination at the Oprk1 gene locus in different brain regions and organs of the mouse using PCR primers specific to the recombined DNA (Figure 1). PCR analyses showed Cre-mediated recombination in the VTA/SN region, and olfactory bulb. All other areas analyzed did not show the recombination band (Figure 1a). In previous studies, we and others have shown that Cre recombinase activity is sufficiently robust to mediate genomic recombination in virtually all DA neurons expressing DAT by crossing the DATCre mice with a lacZ reporter strain (Backman et al, 2006), and several other transgenic strains with loxp-flanked gene loci (Diaz-Ruiz et al, 2009, 2012; Luo et al, 2010; Di et al, 2010;Di Salvio et al, 2010; Bello et al, 2011; Cohen et al, 2012; Zhang et al, 2012). In addition, to further verify the specificity of the mutation in $\mathrm{KOR}^{\text {loxp }}$ mice, we performed double ISH in the SN/VTA region with probes specific for DAT and KOR mRNA. Qualitative analyses performed in 20 random fields of view $(\times 40)$ per animal within the SN/VTA region (WT, $n=3$ and KO, $n=3$ ) showed that DNA recombination in the Oprk1 gene containing loxp sites was specific to all neurons expressing DAT mRNA (Figure $1 \mathrm{~b}$ and $\mathrm{c}$ ), as we did not find DAT-positive neurons expressing KOR mRNA in DATCre-KOR KO mice.

\section{Conditional Deletion of KORs on DA Neurons and Effects on NAc DA and CPA}

To evaluate the role of KORs localized on DA neurons, we developed conditional KO mice with KORs deleted only on 

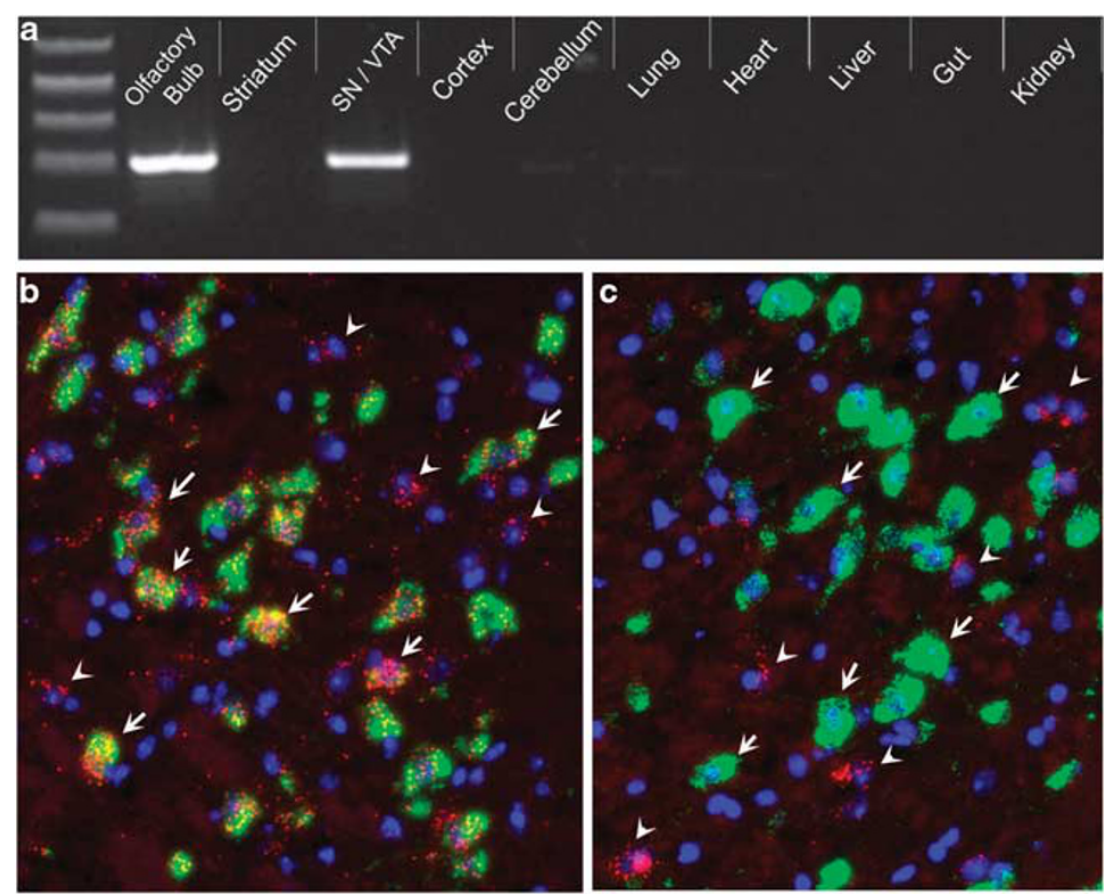

Figure I Cre-mediated recombination of the KOR gene is specific to dopamine transporter mRNA-expressing neurons in the VTA region. (a) PCR screen for Cre-mediated KOR exon 2 deletion. Genomic DNAs were prepared from the indicated tissues from a DATCre-KORflox mouse. PCR analyses show KOR deletion is specific to the olfactory bulb and SN/NTA regions. KOR deletion was not detected in other brain areas or tissues analyzed. ( $b$ and $c$ ) Representative images of dual fluorescence in-situ hybridization of DAT mRNA (green) and KOR mRNA (red) in the VTA of a DATCre-KOR KO (c) and control (b) mouse. Blue signal represents DAPI nucleic acid stain. Arrows indicate cells expressing DAT mRNA. DAT-positive neurons co-express KOR mRNA in WT animals (b). As predicted, DAT-positive neurons do not express KOR mRNA in DAT-KOR KO mice (c). In addition, KOR mRNA was present in DAT-negative neurons in both WT and KO animals (arrowheads in b and c).

DA neurons (DATCre-KOR KO). Figure 2 shows representative images of dual fluorescence ISH of DAT mRNA (green) and KOR mRNA (red) in the VTA of a WT (A) and DATCre-KOR KO (B) mouse. As expected, DAT-positive neurons co-expressed KOR mRNA in WT, but there was no KOR mRNA expression in DAT-positive neurons in DATCre-KOR KO mice.

To assess if WT and DATCre-KOR KO mice have different DA response to systemically administered U69,593, we performed microdialysis experiments (Figure 2c). A one-way repeated-measures ANOVA showed significant drug challenge $\times$ genotype interaction $\left(\mathrm{F}_{(1,8)}=4.4\right.$; $p=0.048)$ and significant difference between baseline and U69,593 challenge in WT $\left(\mathrm{F}_{(1,4)}=13.8 ; p=0.02\right)$, but not in DATCre-KOR KO mice $\left(\mathrm{F}_{(1,4)}=1.2 ; p=0.34\right)$. These results indicate that systemic U69,593 decreased NAc DA overflow in WT mice, but had no effect in DATCre-KOR KO animals.

We next assessed whether KORs localized on DA neurons are necessary for the development of CPA to KOR agonists using the same two lines of mice (Figure $2 \mathrm{~d}$ and e). A twoway repeated-measures ANOVA analysis of the time spent in vehicle- and U69,593-paired compartments during preand post-tests revealed significant interaction between time of the test (before and after) and treatment as well as time of the test $\times$ genotype $\times$ treatment interaction $\left(\mathrm{F}_{(1,44)}=26.4\right.$; $p<0.01$ and $\mathrm{F}_{(1,44)}=11.1 ; p<0.01$, respectively). Furthermore, there was interaction time $\times$ treatment interaction in WT, but not in DATCre-KOR KO animals $\left(\mathrm{F}_{(1,22)}=30.8\right.$; $p<0.01$ and $\mathrm{F}_{(1,44)}=1.9 ; p=0.18$, respectively), indicating that KOR activation caused CPA in WT, but not in KO animals (Figure 2d). Consequently, there was a significant difference between time spent in U69,593-paired compartment during pre-test and time spent in the same compartment during post-test in $\mathrm{WT}\left(\mathrm{F}_{(1,11)}=16.7 ; p<0.01\right)$, but not in DATCre-KOR KO $\left(\mathrm{F}_{(1,11)}=0.9 ; p=0.34\right)$. Similarly, there was significant genotype $\times$ treatment interaction for conditioning scores $\left(\mathrm{F}_{(147)}=11.1 ; p<0.01\right)$ and significant difference between vehicle and U69,593 scores in WT $\left(\mathrm{F}_{(1,23)}=30.8 ; p<0.01\right)$, but not DATCre-KOR KO animals $\left(\mathrm{F}_{(1,23)}=1.9 ; p=0.18\right.$; Figure $\left.2 \mathrm{e}\right)$. In addition, there was a significant genotype effect for conditioning scores in U69,593-paired compartments $\left(\mathrm{F}_{(1,23)}=6.5 ; p=0.018\right)$. Overall, these data indicate that WT mice showed significant U69,593-induced CPA, whereas KO animals did not develop KOR-mediated CPA. Importantly, there was no difference between genotypes in locomotor activity during conditioning phase $\left(\mathrm{F}_{(1,10)}=0.09 ; p=0.92\right)$, as well as during pre$\left(\mathrm{F}_{(1,11)}=0.59 ; p=0.46\right)$ and post- $\left(\mathrm{F}_{(1,11)}=0.53 ; p=0.48\right)$ tests, indicating that the lack of KOR-induced CPA in KO animals is not due to changes in locomotion.

\section{Intra-VTA Expression of KORs in DATCre-KOR KO Mice Permits CPA to U69,593}

To confirm that KORs were expressed on DA neurons following intra-VTA delivery of AAV2-CAG-KOR, we examined the expression of KOR mRNA and its co-localization with the DAT immunohistochemical signal (Figure $3 a$ and $b$ ). We found that KOR mRNA was co-localized with DAT 

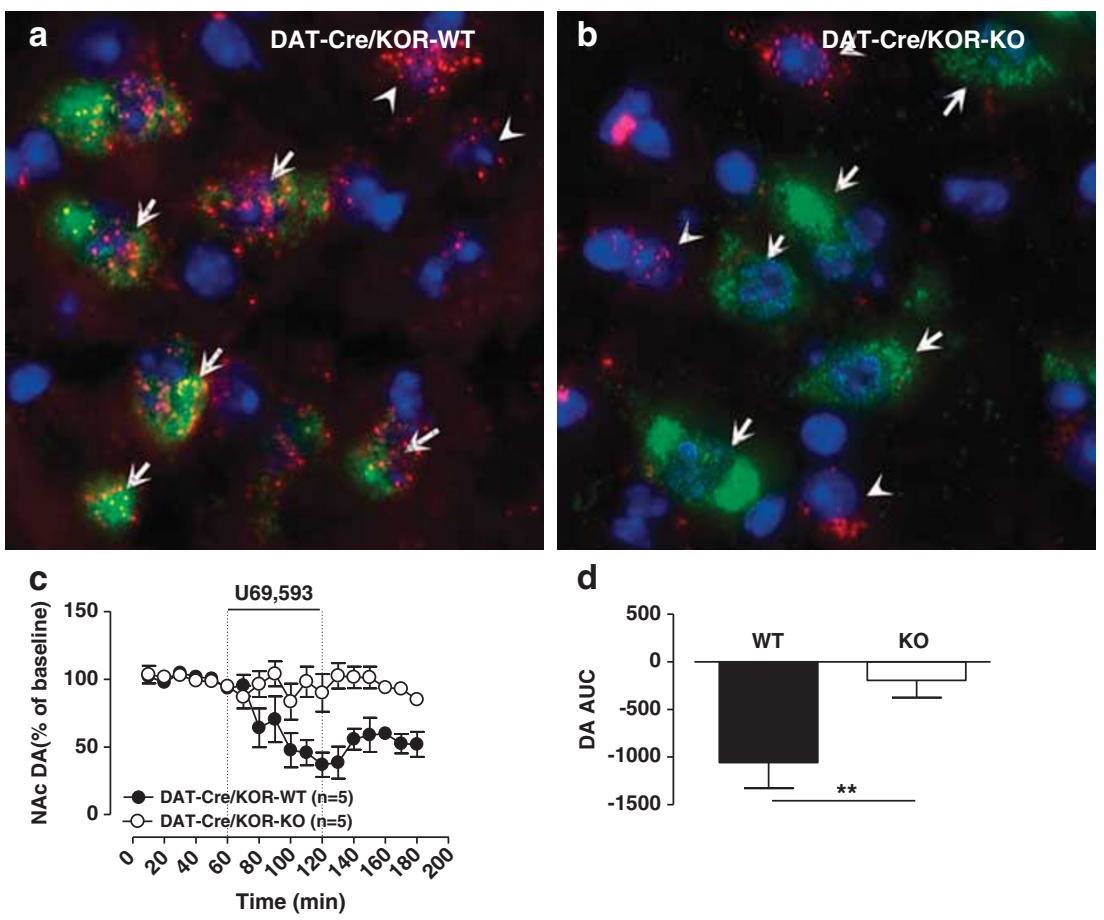

d
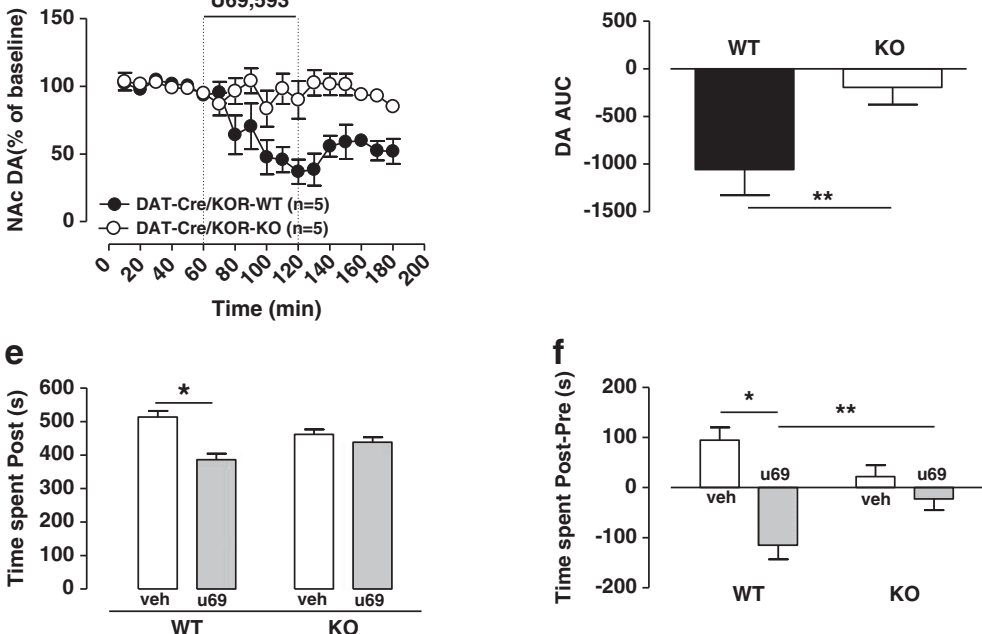

Figure 2 Conditional deletion of KORs on DA neurons abolished NAc DA response to systemically administered KOR agonist U69,593 and development of KOR-mediated CPA. (a and b) Representative images of dual fluorescence in-situ hybridization of DAT mRNA (green) and KOR mRNA (red) in the VTA of a WT (DAT ${ }^{\text {Cre/wt }} \mathrm{KOR}^{\text {wt/wt; }}$ A) and KO (DAT ${ }^{\text {Cre/wt }} K \mathrm{KR}^{\text {loxp/loxp; }}$ B) mouse. Blue signal represents DAPI nucleic acid stain. Arrows indicate cells expressing DAT mRNA. DAT-positive neurons co-express KOR mRNA in control animals (a). As predicted, DAT-positive neurons do not express KOR mRNA in DATCre-KOR KO mice (b). In addition, KOR mRNA was present in DAT-negative neurons in both genotypes (arrowheads in a and b). (c) Time course of basal and U69,593-induced NAc DA overflow in WT (filled circles) and KO (open circles) mice. The data are expressed as mean \% of baseline \pm SEM. (d) AUC values of DA levels after U69,593 challenge in WT (filled bars) and KO (open bars) animals. ** Significant difference between WT and $\mathrm{KO}$ animals. (e) Time spent in vehicle- (open bars) or U-69,593- (filled bars) paired compartments during post-test. The data are expressed as mean of the time spent \pm SEM. *A significant difference between time spent in vehicle- and U69,593-paired compartments. ( $f$ ) Conditioning score defined as time spent in vehicle- or drug-paired place during post-test minus that spent in the same place during post-test. The data are expressed as mean of the time spent \pm SEM. * and *** reflect significant difference between vehicle- and U69,593-paired compartments and between WT and KO animals, respectively.

immunosignal, and in cells that lacked the DAT in the VTA. To assess if intra-VTA viral expression of KORs in DATCreKOR KO mice can restore the effect of U69,593 on DA overflow in NAc, we performed microdialysis experiments 3 weeks after injections of AAV2-CAG-KOR (Figure 3c). A one-way repeated-measures ANOVA showed significant drug challenge $\times$ genotype interaction $\left(\mathrm{F}_{(1,8)}=7.6 ; p=0.025\right)$ and significant difference between baseline and U69,593 challenge in AAV2-CAG-KOR $\left(\mathrm{F}_{(1,4)}=129 ; p<0.01\right)$, but not in control mice injected with AAV2-CAG-GFP $\left(\mathrm{F}_{(1,4)}=\right.$ $2.1 ; p=0.22)$. These results indicate that intra-VTA microinjection of AAV2-CAG-KOR permitted the inhibition of DA overflow by U69,593 in the NAc of DATCre-KOR KO animals.

To confirm the necessity of KORs on DA neurons for development of KOR-mediated CPA, we attempted to rescue this function by restoring KORs on DA neurons.
Conditioning experiments started following 3 weeks of AAV2 delivery (see Figure $3 c$ and d). Two-way repeatedmeasures ANOVA analysis of time spent in vehicle- and U69,593-paired compartments during pre- and post-tests revealed significant time of the test (before and after) $\times$ microinjections $\times$ conditioning as well microinjections $\times$ conditioning interactions $\left(\mathrm{F}_{(1,44)}=5.8 ; p=0.02\right.$ and $\mathrm{F}_{(1,44)}=5.7 ; p=0.021$, respectively). Consequently, there was a significant effect of conditioning in AAV2-CAG-KOR$\left(\mathrm{F}_{(1,23)}=15.3 ; p=0.01\right)$, but not in AAV2-CAG-GFP-injected animals $\left(\mathrm{F}_{(1,23)}=0.1 ; p=0.76\right.$; Figure $\left.3 \mathrm{c}\right)$. Similarly, when conditioned scores were analyzed, there was significant microinjections $\times$ conditioning interaction $\quad\left(\mathrm{F}_{(147)}=5.85\right.$; $p=0.02)$ and significant effect of conditioning in AAV2CAG-KOR-injected animals $\left(\mathrm{F}_{(1,23)}=14.9 ; p=0.01\right)$ as well significant effect of microinjections for U69,593-paired compartments $\left(\mathrm{F}_{(1,23)}=4.02 ; p=0.05\right.$; Figure 3d). Overall, 

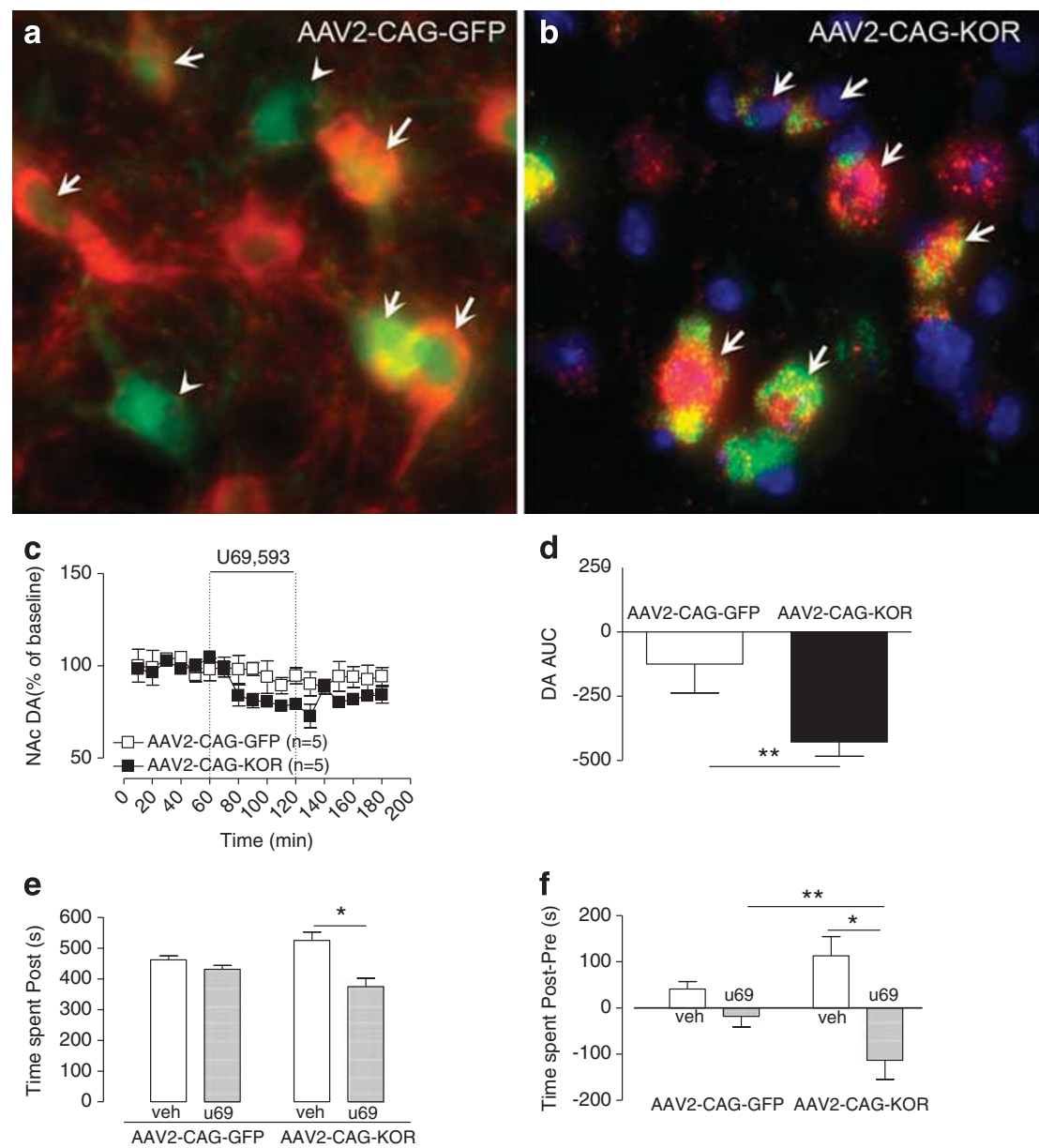

Figure 3 Viral rescue of KOR function in DATCre-KOR KO mice VTA restored NAc DA response to KOR agonist and KOR-mediated CPA. ( $a$ and $b$ ) Representative images of the VTA region in DATCre-KOR KO mice injected with AAV2-CAG-GFP (a) or AAV2-CAG-KOR (b) viral vectors, (a) Arrows show DAT immuno-stained cells (red) co-expressing GFP. GFP was also expressed in DAT-negative neurons (arrowheads). (b) Arrows show KOR mRNA signal (red probe) co-localized with DAT mRNA signal (green probe). Blue signal represents DAPI nucleic acid stain. (c) Time course of basal and U69,593-induced NAc DA overflow in AAV2-CAG-GFP- (open squares) and AAV2-CAG-KOR-injected (filled squares) mice. The data are expressed as mean \% of baseline \pm SEM. (d) AUC values of DA levels after U69,593 challenge in AAV2-CAG-GFP- (filled bars) and AAV2-CAG-KOR-injected (open bars) animals. ** Significant difference between AAV2-CAG-GFP and AAV2-CAG-KOR groups. (e) Time spent in vehicle- (open bars) or U-69,593- (filled bars) paired compartments during post-test. The data are expressed as mean of the time spent \pm SEM. *A significant difference between time spent in vehicle- and U69,593-paired compartments. (f) Conditioning score defined as time spent in vehicle- or drug-paired place during post-test minus that spent in the same place during post-test. The data are expressed as mean of the time spent \pm SEM. * and ** reflect a significant difference between vehicle- and U69,593-paired compartments and between AAV2-CAG-GFP- and AAV2-CAG-KOR-injected animals, respectively.

these data indicate that following intra-VTA microinjections of AAV2-CAG-KOR, DATCre-KOR KO mice developed U69,593-induced CPA, whereas following intra-VTA microinjections of AAV2-CAG-GFP they were not able to develop KOR-mediated CPA.

\section{DISCUSSION}

We have shown that DATCre-KOR KO mice, lacking functional KORs in DA cells, fail to develop a CPA to the KOR agonist U69,593. Furthermore, the inhibition of DA overflow in the NAc produced by KOR activation is also lacking in these mice. We also demonstrate that U69,593induced CPA and inhibition of NAc DA overflow can be reversed in DATCre-KOR KO mice following intra-VTA expression of KORs via AAV-mediated gene delivery. Together, these results support the hypothesis that KORs on midbrain DA neurons are necessary for KOR-mediated place aversion.

Extensive evidence indicates that KOR agonists produce aversion and negative effect in rodents (Mucha and Herz, 1985; Carlezon Jr, et al, 2006; Land et al, 2009), and psychotomimetic and anxiogenic effects in humans (Pfeiffer et al, 1986). There is also compelling evidence that DA neurotransmission is regulated by KORs and that the aversive effects of KOR agonists are mediated via the suppression of DA in the NAc (Bals-Kubik et al, 1993b; Shippenberg et al, 1992; Spanagel et al, 1994; Shippenberg and Elmer, 1998). Thus, the KOR system exerts a tonic control on DA release and uptake in the NAc (Heijna et al, 1990; Spanagel et al, 1992; Thompson et al, 2000; Chefer et al, 2005), and this is consistent with the high expression of KORs observed in the region, including on DA axon terminals (Svingos et al, 2001). Systemic administration of KOR agonists can also influence DA neurotransmission via 
direct inhibition of VTA DA neurons projecting to prefrontal cortex (Margolis et al, 2006). However, not all aversive events are associated with a decrease in dopaminergic activity (Horvitz, 2000), and inhibition of DA release may not be the only KOR action relevant to its aversive action. In this regard, recent studies have shown that KOR agonists can inhibit glutamatergic and GABAergic synaptic transmission in the NAc shell (Hjelmstad and Fields, 2001; Hjelmstad and Fields, 2003), which may contribute to the KOR-mediated aversion. These results are in accordance with previous anatomical data demonstrating that $21 \%$ of KOR-immunoreactive terminals in the shell form excitatory type synapses (Svingos et al, 1999), as well as in vivo data showing that KORs regulate a calciumdependent component of the striatum glutamate influx induced by glutamate reuptake blocker, 1-trans-pyrrolidine2,4-dicarboxylate (Rawls and McGinty, 1998).

More recent findings, on the other hand, implicate KORs in the serotonergic DRN as being both necessary and sufficient to mediate aversive behavioral responses (Land et al, 2009). In Land et al (2009) study, it was reported that DA-deficient mice developed CPA to the KOR agonist U50,488, implying decreased DA release caused by KOR agonists may not be necessary for CPA. However, whereas the role of the serotonergic projection from the DRN to the NAc in CPA is undisputable, this study did not rule out a role for DA in this behavior. Thus, all constitutive KOs may exhibit compensatory neurobiological adaptations during development that may alter the balance of the monoaminergic systems that are involved in CPA. Moreover, the DA-deficient mice used in the study by Land et al (2009) were hypoactive, which necessitated the use of the adenosine receptor antagonist/calcium releaser caffeine before CPA tests, which complicates interpretation of their data.

Recently, two studies (Tejeda et al, 2013; Van't Veer et al, 2013) used conditional KOR KOs in DA-containing neurons similar to the present study. The paper by Tejeda et al (2013) focused specifically on KORs located on dopaminergic terminals in the mPFC. DAT KOR KO mice were used in that study in order to elucidate if inhibition of DA by intramPFC U69,593 is directly mediated by KORs located on DA varicosities in the MPFC and not by indirect circuitry. It has been shown that mPFC DA uptake and release were unaltered in these mice relative to controls, but attenuation of mPFC DA overflow by local U69,593 was not observed due to lack of KORs on DA varicosities. (Van't Veer et al, 2013) conducted detailed behavioral characterization of this line. The main behavioral difference from controls reported by these authors was a reduction of anxiety-like behaviors: a decreased latency to enter the lit side of a light-dark box and a preference for the center of an open field box. However, they report no differences in total time in the light side of the light-dark box and no differences in general locomotion. Moreover, they report no differences in anxiety-like behaviors as assessed on an elevated plus-maze. In addition, DAT-KOR KOs had increased sensitivity to cocaine in accord with a role of KOR in negative regulation of DA neurotransmission. Taken together, ablation of KORs in DA neurons had an anxiolytic-like effect, consistent with previously reported effects of KOR antagonists (for review, see Carlezon Jr et al (2009) and Carroll and Carlezon Jr (2013)).
It is noteworthy to say that the above papers did not address the question of necessity of KORs located on DA neurons for aversive behavioral, although Tejeda et al (2013) have shown that mPFC KOR signaling was necessary for KOR-mediated aversion. With this recently published work and the current study as evidence, it appears that both MPFC and NAc are necessary for KOR-mediated CPA. Along these lines, we can infer that perhaps the same will be true for other DA projection sites. Future studies should focus on investigating additional DA projection sites to further determine the role of DA in KOR-mediated CPA.

The results of the present study demonstrate that specific KOR deletion on DA neurons disrupts behavioral and neurochemical responses typically observed after systemic administration of KOR agonists. Thus, DATCre-KOR KO animals did not develop CPA to U69,593, and reduction of DA levels in the NAc was absent after systemic administration of U69,593. Moreover, restoration of KORs in the VTA resulted in phenotypic reinstatement of both the neurochemical and behavioral responses to KOR agonist in DATCre-KOR KO animals. Therefore, these results support the hypothesis that activation of KORs on DA neurons is necessary for KOR-mediated place aversion, because generally a condition $\mathrm{A}$ is necessary for a condition $\mathrm{B}$, if the nonexistence of $\mathrm{A}$ brings about the nonexistence of B. Moreover, an assumption that KORs on DA neurons are sufficient for KOR-induced CPA can be made only if restoring KORs on DA neurons in constitutive KOR KOs lacking KORs would restore CPA. It would be reasonable to address the issue of sufficiency in future studies.

Taken together with recent findings regarding possible contribution of serotonergic (Land et al, 2009) as well as glutamatergic and GABAergic neurotransmission (Hjelmstad and Fields, 2001; Hjelmstad and Fields, 2003), our results may suggest that the behavioral effects of KOR activation involves interaction of multiple neurotransmitters converging on GABAergic medium spiny neurons in the mesolimbic system, and further studies examining a unique role for each of them as well as possible mechanisms of their interactions are warranted.

\section{FUNDING AND DISCLOSURE}

The authors declare no conflict of interest.

\section{ACKNOWLEDGEMENTS}

This work was supported by the National Institute on Drug Abuse Intramural Research Program. We thank Dr Carl Lupica for his helpful editorial comments and suggestions; and Dr Jennifer Whistler for providing KOR ${ }^{\text {loxp }}$ mice.

\section{Author contributions}

V.C. and T.S.S. designed the research; C.M.B. created conditional knock-outs; V.C., E.D.G., and C.M.B. performed the research; V.C. and E.D.G. analyzed the data; V.C., E.D.G., and C.M.B. wrote the paper. 


\section{REFERENCES}

Backman CM, Malik N, Zhang Y, Shan L, Grinberg A, Hoffer BJ et al (2006). Characterization of a mouse strain expressing Cre recombinase from the 3 ' untranslated region of the dopamine transporter locus. Genesis 44: 383-390.

Bals-Kubik R, Ableitner A, Herz A, Shippenberg TS (1993a). Neuroanatomical sites mediating the motivational effects of opioids as mapped by the conditioned place preference paradigm in rats. J Pharmacol Exp Ther 264: 489-495.

Bals-Kubik R, Ableitner A, Herz A, Shippenberg TS (1993b). Neuroanatomical sites mediating the motivational effects of opioids as mapped by the conditioned place preference paradigm in rats. J Pharmacol Exp Ther 264: 489-495.

Bello EP, Mateo Y, Gelman DM, Noain D, Shin JH, Low MJ et al (2011). Cocaine supersensitivity and enhanced motivation for reward in mice lacking dopamine D2 autoreceptors. Nat Neurosci 14: 1033-1038.

Carlezon WA Jr, Beguin C, Dinieri JA, Baumann MH, Richards MR, Todtenkopf MS et al (2006). Depressive-like effects of the \{kappa\}-opioid receptor agonist salvinorin A on behavior and neurochemistry in rats. J Pharmacol Exp Ther 316: 440-447.

Carlezon WA Jr, Beguin C, Knoll AT, Cohen BM (2009). Kappaopioid ligands in the study and treatment of mood disorders. Pharmacol Ther 123: 334-343.

Carroll FI, Carlezon WA Jr (2013). Development of kappa opioid receptor antagonists. J Med Chem 56: 2178-2195.

Chefer VI, Czyzyk T, Bolan EA, Moron J, Pintar JE, Shippenberg TS (2005). Endogenous kappa-opioid receptor systems regulate mesoaccumbal dopamine dynamics and vulnerability to cocaine. J Neurosci 25: 5029-5037.

Chefer VI, Denoroy L, Zapata A, Shippenberg TS (2009). Mu opioid receptor modulation of somatodendritic dopamine overflow: GABAergic and glutamatergic mechanisms. Eur J Neurosci 30: $272-278$.

Chefer VI, Kieffer BL, Shippenberg TS (2004). Contrasting effects of mu opioid receptor and delta opioid receptor deletion upon the behavioral and neurochemical effects of cocaine. Neuroscience 127: 497-503.

Chefer VI, Zapata A, Shippenberg TS, Bungay PM (2006). Quantitative no-net-flux microdialysis permits detection of increases and decreases in dopamine uptake in mouse nucleus accumbens. J Neurosci Methods 155: 187-193.

Cohen JY, Haesler S, Vong L, Lowell BB, Uchida N (2012). Neurontype-specific signals for reward and punishment in the ventral tegmental area. Nature 482: 85-88.

Cunningham CL, Gremel CM, Groblewski PA (2006). Druginduced conditioned place preference and aversion in mice. Nat Protoc 1: 1662-1670.

Di Salvio M., Di Giovannantonio LG, Acampora D, Prosperi R, Omodei D, Prakash N et al (2010). Otx2 controls neuron subtype identity in ventral tegmental area and antagonizes vulnerability to MPTP. Nat Neurosci 13: 1481-1488.

Di SM, Di Giovannantonio LG, Acampora D, Prosperi R, Omodei D, Prakash N et al (2010). Otx2 controls neuron subtype identity in ventral tegmental area and antagonizes vulnerability to MPTP. Nat Neurosci 13: 1481-1488.

Diaz-Ruiz O, Zapata A, Shan L, Zhang Y, Tomac AC, Malik N et al (2009). Selective deletion of PTEN in dopamine neurons leads to trophic effects and adaptation of striatal medium spiny projecting neurons. PLoS One 4: e7027.

Diaz-Ruiz O, Zhang Y, Shan L, Malik N, Hoffman AF, Ladenheim B et al (2012). Attenuated response to methamphetamine sensitization and deficits in motor learning and memory after selective deletion of beta-catenin in dopamine neurons. Learn Mem 19: 341-350.

Heijna MH, Padt M, Hogenboom F, Portoghese PS, Mulder AH, Schoffelmeer AN (1990). Opioid receptor-mediated inhibition of dopamine and acetylcholine release from slices of rat nucleus accumbens, olfactory tubercle and frontal cortex. Eur $J$ Pharmacol 181: 267-278.

Hjelmstad GO, Fields HL (2001). Kappa opioid receptor inhibition of glutamatergic transmission in the nucleus accumbens shell. J Neurophysiol 85: 1153-1158.

Hjelmstad GO, Fields HL (2003). Kappa opioid receptor activation in the nucleus accumbens inhibits glutamate and GABA release through different mechanisms. J Neurophysiol 89: 2389-2395.

Horvitz JC (2000). Mesolimbocortical and nigrostriatal dopamine responses to salient non-reward events. Neuroscience 96: 651-656.

Knoll AT, Muschamp JW, Sillivan SE, Ferguson D, Dietz DM, Meloni EG et al (2011). Kappa opioid receptor signaling in the basolateral amygdala regulates conditioned fear and anxiety in rats. Biol Psychiatry 70: 425-433.

Land BB, Bruchas MR, Schattauer S, Giardino WJ, Aita M, Messinger D et al (2009). Activation of the kappa opioid receptor in the dorsal raphe nucleus mediates the aversive effects of stress and reinstates drug seeking. Proc Natl Acad Sci USA 106: 19168-19173.

Lubow RE (1973). Latent inhibition. Psychol Bull 79: 398-407.

Luo Y, Good CH, Diaz-Ruiz O, Zhang Y, Hoffman AF, Shan L et al (2010). NMDA receptors on non-dopaminergic neurons in the VTA support cocaine sensitization. PLoS One 5: e12141.

Margolis EB, Lock H, Chefer VI, Shippenberg TS, Hjelmstad GO, Fields HL (2006). \{kappa\} opioids selectively control dopaminergic neurons projecting to the prefrontal cortex. Proc Natl Acad Sci USA 103: 2938-2942.

Mucha RF, Herz A (1985). Motivational properties of kappa and mu opioid receptor agonists studied with place and taste preference conditioning. Psychopharmacology (Berl) 86: 274-280.

Paxinos G, Franklin KBJ (2001). The mouse brain in stereotaxic coordinates. Academic Press, $350 \mathrm{p}$.

Pfeiffer A, Brantl V, Herz A, Emrich HM (1986). Psychotomimesis mediated by kappa opiate receptors. Science 233: 774-776.

Rawls SM, McGinty JF (1998). Kappa receptor activation attenuates L-trans-pyrrolidine-2,4-dicarboxylic acid-evoked glutamate levels in the striatum. J Neurochem 70: 626-634.

Shippenberg TS, Bals-Kubik R, Herz A (1993). Examination of the neurochemical substrates mediating the motivational effects of opioids: role of the mesolimbic dopamine system and D-1 vs. D-2 dopamine receptors. J Pharmacol Exp Ther 265: 53-59.

Shippenberg TS, Elmer GI (1998). The neurobiology of opiate reinforcement. Crit Rev Neurobiol 12: 267-303.

Shippenberg TS, Herz A, Spanagel R, Bals-Kubik R, Stein C (1992). Conditioning of opioid reinforcement: neuroanatomical and neurochemical substrates. Ann N Y Acad Sci 654: 347-356.

Spanagel R, Almeida OF, Bartl C, Shippenberg TS (1994). Endogenous kappa-opioid systems in opiate withdrawal: role in aversion and accompanying changes in mesolimbic dopamine release. Psychopharmacology (Berl) 115: 121-127.

Spanagel R, Herz A, Shippenberg TS (1992). Opposing tonically active endogenous opioid systems modulate the mesolimbic dopaminergic pathway. Proc Natl Acad Sci USA 89: 2046-2050.

Svingos AL, Chavkin C, Colago EE, Pickel VM (2001). Major coexpression of kappa-opioid receptors and the dopamine transporter in nucleus accumbens axonal profiles. Synapse 42: 185-192.

Svingos AL, Colago EE, Pickel VM (1999). Cellular sites for dynorphin activation of kappa-opioid receptors in the rat nucleus accumbens shell. J Neurosci 19: 1804-1813.

Tejeda HA, Counotte DS, Oh E, Ramamoorthy S, Schultz-Kuszak KN, Backman CM et al (2013). Prefrontal cortical kappa-opioid receptor modulation of local neurotransmission and conditioned place aversion. Neuropsychopharmacology 38: 1770-1779. 
Thompson AC, Zapata A, Justice JB, Vaughan RA, Sharpe LG, Shippenberg TS (2000). kappa-Opioid receptor activation modifies dopamine uptake in the nucleus accumbens and opposes the effects of cocaine. J Neurosci 20: 9333-9340.

Van't Veer A, Bechtholt AJ, Onvani S, Potter D, Wang Y, Liu-Chen LY et al (2013). Ablation of kappa-opioid receptors from brain dopamine neurons has anxiolytic-like effects and enhances cocaine-induced plasticity. Neuropsychopharmacology 38: 1585-1597.
Zhang Y, Butelman ER, Schlussman SD, Ho A, Kreek MJ (2005). Effects of the plant-derived hallucinogen salvinorin A on basal dopamine levels in the caudate putamen and in a conditioned place aversion assay in mice: agonist actions at kappa opioid receptors. Psychopharmacology (Berl) 179: 551-558.

Zhang Y, Granholm AC, Huh K, Shan L, Diaz-Ruiz O, Malik N et al (2012). PTEN deletion enhances survival, neurite outgrowth and function of dopamine neuron grafts to MitoPark mice. Brain 135: 2736-2749. 